\title{
Autoantibodies are present in the bronchoalveolar lavage but not circulation in patients with fibrotic interstitial lung disease
}

\author{
Karim Boustani ${ }^{1,2}$, Poonam Ghai ${ }^{1}$, Rachele Invernizzi $\mathbb{1}^{1}$, Richard J. Hewitt ${ }^{1,3}$, Toby M. Maher ${ }^{1,3,4}$, \\ Quan-Zhen Li ${ }^{5}$, Philip L. Molyneaux $\mathbb{1}^{1,3,6}$ and James A. Harker (D) ${ }^{1,2,6}$
}

${ }^{1}$ National Heart and Lung Institute, Imperial College London, London, UK. ${ }^{2}$ Asthma UK Centre in Allergic Mechanisms of Asthma, London, UK. ${ }^{3}$ Royal Brompton Hospital, London, UK. ${ }^{4}$ Hastings Centre for Pulmonary Research and Division of Pulmonary, Critical Care and Sleep Medicine, Keck School of Medicine, University of Southern California, Los Angeles, CA, USA. ${ }^{5}$ Dept of Immunology and Internal Medicine, Microarray and Immune Phenotyping Core Facility, University of Texas Southwestern Medical Centre, Dallas, TX, USA. ${ }^{6}$ Denotes equal contribution.

Corresponding author: James A. Harker (j.harker@imperial.ac.uk)

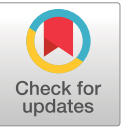

Copyright $\odot$ The authors 2022

This version is distributed under the terms of the Creative Commons Attribution NonCommercial Licence 4.0. For commercial reproduction rights and permissions contact permissions@ersnet.org

This article has supplementary material available from openres.ersjournals.com

Received: 27 July 2021

Accepted: 23 Oct 2021

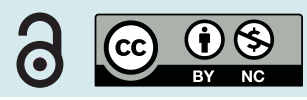

Shareable abstract (@ERSpublications)

Autoantibodies are present in the bronchoalveolar lavage but not circulation in patients with fibrotic interstitial lung disease https://bit.ly/3CNvKjj

Cite this article as: Boustani $\mathrm{K}$, Ghai $\mathrm{P}$, Invernizzi $\mathrm{R}$, et al. Autoantibodies are present in the bronchoalveolar lavage but not circulation in patients with fibrotic interstitial lung disease. ERJ Open Res 2022; 8: 00481-2021 [DOI: 10.1183/23120541.00481-2021].

\section{Abstract}

Background Fibrotic interstitial lung disease (fILD) has previously been associated with the presence of autoantibody. While studies have focused on systemic autoimmunity, the role of local autoantibodies in the airways remains unknown. We therefore extensively characterised the airway and peripheral autoantibody profiles in patients with fILD, and assessed association with disease severity and outcome.

Methods Bronchoalveolar lavage (BAL) fluid was collected from a cohort of fILD patients and total BAL antibody concentrations were quantified. An autoantigen microarray was used to measure IgG and IgA autoantibodies against 122 autoantigens in BAL from 40 idiopathic pulmonary fibrosis (IPF), 20 chronic hypersensitivity pneumonitis (CHP), 20 connective tissue disease-associated ILD (CTD-ILD) patients and 20 controls.

Results A subset of patients with fILD but not healthy controls had a local autoimmune signature in their BAL that was not present systemically, regardless of disease. The proportion of patients with IPF with a local autoantibody signature was comparable to that of CTD-ILD, which has a known autoimmune pathology, identifying a potentially novel subset of patients. The presence of an airway autoimmune signature was not associated with reduced survival probability or changes in lung function in the cohort as a whole. Patients with IPF had increased BAL total IgA and IgG $_{1}$ while subjects with CHP had increased BAL IgA, IgG 1 and IgG $_{4}$. In patients with CHP, increased BAL total IgA was associated with reduced survival probability.

Conclusion Airway autoantibodies that are not present systemically identify a group of patients with fILD and the mechanisms by which these autoantibodies contribute to disease requires further investigation.

\section{Introduction}

Interstitial lung disease (ILD) is an umbrella term for a group of devastating, chronic lung diseases including idiopathic pulmonary fibrosis (IPF), chronic hypersensitivity pneumonitis (CHP) and connective tissue disease-associated ILD (CTD-ILD) [1]. Each of these diseases has a unique aetiology and in the case of IPF, which is the most common form of ILD, the cause remains unknown. This is a growing problem in the UK, with $>5000$ cases diagnosed annually and, despite therapy, a 5-year survival of $\sim 20 \%$ [2]. Despite differing aetiologies and triggers, there is growing evidence of shared pathogenesis across the spectrum of fibrotic ILD (fILD). These diseases arise in response to microinjuries to the respiratory epithelium, which trigger an aberrant wound healing response in genetically susceptible older individuals. The immune system is known to play a role in the pathogenesis of both CHP, largely driven by known environmental antigens, and CTD-ILD, driven by self antigens. The role of the immune system in the 
pathogenesis of IPF is, however, less clear. Recent work has highlighted changes in the lung immune response, especially within the macrophage population, and their correlation with disease outcome, but a source of antigen stimulation has yet to be identified [3].

Autoimmunity occurs as a result of a breakdown of tolerance within the adaptive immune cell compartment, resulting in the generation of antibodies by plasma cells, which target self antigens. The presence of circulating autoantibodies is used to diagnose CTD-ILD clinically. These circulating autoantibodies are often against nuclear components, such as Ro, La, Jo1, Scl70 and double-stranded DNA [4-6]. Associations between circulating autoantibodies and disease outcome are not, however, limited to CTD-ILD; and other forms of fILD, such as CHP, as well as other chronic lung diseases, such as chronic obstructive pulmonary disease (COPD), have been shown to have similar associations [6-10]. Supporting a role for antibody-mediated immunity in the pathogenesis of some patients with pulmonary fibrosis, rituximab, a monoclonal antibody that specifically depletes B-cells, has also been shown to be beneficial in a subset of patients with either CTD-ILD or CHP [11-13]. Much less is known, however, about the contribution of the antibody response to IPF, although a small-scale trial of rituximab in combination with plasmapheresis showed some benefit during acute exacerbations of IPF [14]. Further evidence for humoral dysregulation being a contributor to disease pathology comes from the identification of multiple circulating autoantibodies against various components of alveolar epithelial cells, extracellular matrix components such as collagens I, III and IV, and other lung-specific proteins such as BPIFB1 [5-7, 15-21]. It is not well understood how these autoantibodies drive pathology but autoantibodies targeting proteins expressed almost exclusively in the lung parenchyma, such as KCNRG and BPIFB1, have been identified in patients with fibrotic lung disease [18, 22, 23]. This suggests that autoantibodies against proteins expressed in lung tissue may induce damage and, subsequently, inflammation and aberrant wound repair resulting in fibrosis.

In this study, we sought to determine whether there is a local autoimmune signature in the airways of patients with fILD and whether the presence of airway autoantibodies could be used to predict disease outcome. We demonstrate that there is a significant increase in bronchoalveolar lavage (BAL) fluid IgA and IgG1 in IPF, and increased IgA and IgG $_{4}$ in CHP. A subset of patients with fILD had distinct autoantibody profiles in BAL, the presence of which was not associated with autoantibodies in the circulation. These findings present a novel observation that there is a distinct airway autoantibody signature in a subset of patients with fILD that is not present in healthy individuals. Further work will be required to assess the implications of these findings in clinical practice and elucidate the pathological role airway autoantibodies may play.

\section{Methods}

Patients and sampling

Patients undergoing routine diagnostic bronchoscopy at the Interstitial Lung Disease Unit at the Royal Brompton Hospital (London, UK) between May 2014 and December 2019 were recruited prospectively. The study was approved by the East London and the City Research Ethics Committee (15-LO-1399) and the South Central Hampshire Research Ethics Committee (15/SC/0101). Written informed consent was obtained from all subjects. Subjects with histories of upper or lower respiratory tract infections, antibiotic use in the prior 3 months or history of acute exacerbations were excluded. Bronchoscopy with BAL was performed as described previously [24, 25].

\section{BAL processing}

BAL samples were passed through a $70-\mu \mathrm{m}$ sterile strainer and centrifuged $\left(700 \times \mathrm{g}\right.$ for $5 \mathrm{~min}$ at $4^{\circ} \mathrm{C}$ ). Supernatants were stored at $-80^{\circ} \mathrm{C}$ for further use.

\section{Enzyme-linked immunosorbent assays}

Total antibody ELISAs for IgA, IgG, IgM, IgG 1 , IgG 2 , IgG 3 and $\operatorname{IgG}_{4}$ were carried out on BAL supernatants according to the manufacturer's instructions (Invitrogen, UK). Vitronectin- and collagen V-specific ELISAs were developed based on a standard sandwich ELISA assay. Plates were coated with either $0.625 \mu \mathrm{g} \cdot \mathrm{mL}^{-1}$ recombinant human vitronectin (PromoCell, Germany) or $0.1 \mu \mathrm{g} \cdot \mathrm{mL}^{-1}$ human collagen V (Sigma-Aldrich, UK). Serial dilutions of BAL and plasma were performed starting at neat for BAL and 1:20 for plasma. Plates were incubated with horseradish peroxidase-conjugated anti-human IgG or IgA antibodies at a 1:4000 dilution (SouthernBiotech, USA) and developed with 3,3',5,5'-tetramethylbenzidine. Absorbance at $450 \mathrm{~nm}$ was measured using a SpectraMax i3x plate reader (Molecular Devices, USA). 


\section{Autoantigen array}

IgA and IgG autoantibody reactivities against a panel of 122 autoantigens were measured in $50 \mu \mathrm{L}$ BAL using an array developed by the University of Texas Southwestern Medical Center (table S1) [8, 26]. Briefly, samples were incubated with autoantigens (listed in table S1) printed on 16-pad FAST ${ }^{\mathrm{TM}}$ microarray slides before being incubated with secondary fluorophore-conjugated anti-human IgG or IgA antibodies. Following detection, images were analysed by Genepix Pro 6.0 (Molecular Devices, USA). Analysed data underwent quality control that included filtering bad spot data and batch effect correction. Average net fluorescence intensities (NFIs) and signal/noise ratios (SNRs) were calculated by subtracting negative control (PBS) fluorescence values from sample fluorescence values. If $\mathrm{SNR} / \mathrm{NFI}<0, \mathrm{SNR} / \mathrm{NFI}$ was set to 0.001; if $\mathrm{NFI}<20$ and $\mathrm{SNR}>5$, then SNR=NFI $\cdot 0.15$; and if $\mathrm{SNR}<0.05$ and NFI $>20$, then NFI was set to 0.001 . To avoid outliers in either the NFI or SNR values, antibody scores were calculated (Ab score $=\log _{2}(\mathrm{NFI} \cdot \mathrm{SNR}+1)$. All data were normalised using variance stabilising normalisation.

\section{Statistical analysis}

Nonparametric Mann-Whitney U-tests for two groups or Kruskal-Wallis tests with Dunn's multiple comparisons for more than two groups were performed using GraphPad Prism. Kaplan-Meier analysis was used to compare mortality between subjects with or without airway autoantibodies. Both univariate and multivariate Cox proportional hazard regression analyses were carried out in R. Heatmaps were generated using Morpheus, and columns and rows were clustered using the one minus Pearson correlation method (Broad Institute, USA).

Results

$\operatorname{lgA}, \lg \mathrm{G}_{1}$ and $\lg \mathrm{G}_{4}$ immunoglobulins are increased in IPF and CHP BAL

Given that previous studies have shown alterations in both circulating and airway total immunoglobulins in fILD, we first investigated whether immunoglobulin concentrations were elevated in patients with IPF and CHP compared to healthy controls [15]. A significant increase in serum IgG, but not IgA or IgM, was observed in patients with IPF compared to healthy controls (figure 1a). In the BAL, we observed a statistically significant increase in IgA in both patients with IPF and CHP compared to healthy controls, as well as an increase in IgG in patients with CHP (figure 1b). There also appeared to be a trend toward increase in IgG in patients with IPF compared to healthy controls (figure 1b). Analysis of IgG isotypes showed that $\operatorname{IgG}_{1}$ and $\operatorname{IgG}_{4}$ concentrations were significantly increased in patients with $\mathrm{CHP}$, and $\operatorname{IgG}_{1}$ was also significantly increased in patients with IPF compared to healthy controls (figure 1c). Taken together, these data suggest that increases in BAL IgG and IgA are features of both IPF and CHP.

We performed additional analysis to determine whether increases in BAL immunoglobulins were associated with disease severity and predicted survival. Although there were weak negative correlations between BAL immunoglobulin and lung function in patients with IPF and CHP, this did not influence predicted survival (figure S1A-C). However, in patients with CHP, higher BAL IgA concentrations were associated with significantly reduced survival probability $(p=0.0082)$ (figure S1D). Collectively, these data suggest that BAL immunoglobulin concentrations may be associated with poorer disease outcome in some forms of fILD.

\section{A distinct BAL autoimmune signature is present in a subset of patients with fILD}

Because IgA and IgG were increased in the BAL, we hypothesised this could be linked to increased IgA and IgG autoantibodies in the BAL of patients with fILD. Hence, we performed IgG and IgA autoantibody arrays on 100 BAL samples, which included patients with IPF $(n=40)$, CHP $(n=20)$ and CTD $(n=20)$ as well as healthy and non-disease controls $(n=20)$. Demographic and clinical features are shown in table 1 . Notably, we were able to identify two distinct subsets of patients, those with detectable BAL IgG and IgA autoantibodies against a range of nuclear-, extracellular matrix- and epithelium-associated proteins by hierarchical clustering (figure 2a and b). The broad autoantibody signature was also detectable in two nondisease controls, one of whom had COPD while the other was a patient with bronchiectasis (figure 2a and b). Both of these conditions have previously been associated with the presence of pulmonary autoantibodies and those samples taken from healthy patients had no detectable autoantibodies [8, 9, 27-29].

We next asked whether the patients with increased IgG autoantibodies were also those with increased IgA autoantibodies. We observed a statistically significant correlation between IgG and IgA autoantibody scores, confirming patients who had IgG autoantibody also had IgA autoantibody (figure 2c). Approximately half of the patients with IPF, CHP and CTD had a detectable autoantibody signature in their airways (figure 2d). 

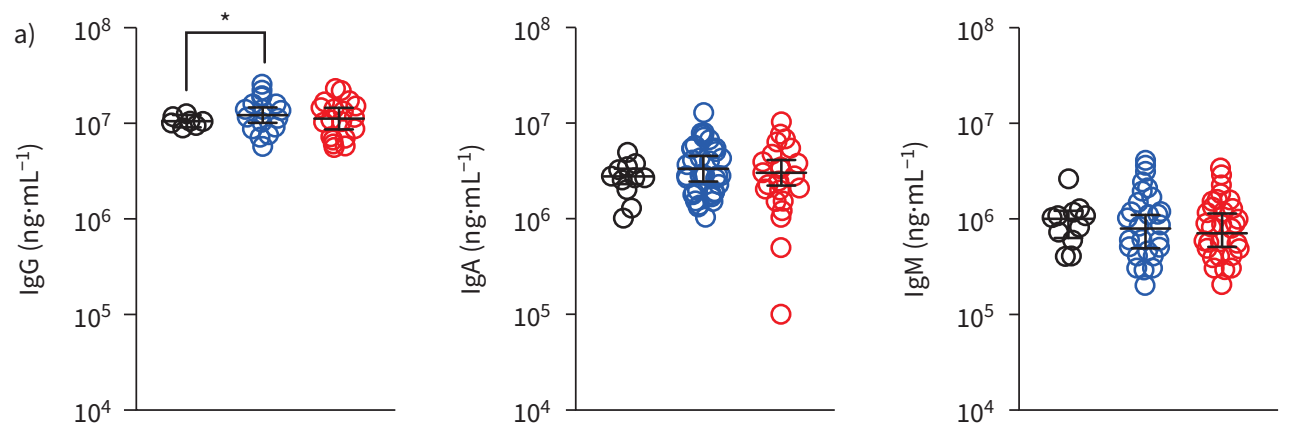

O Control

O IPF
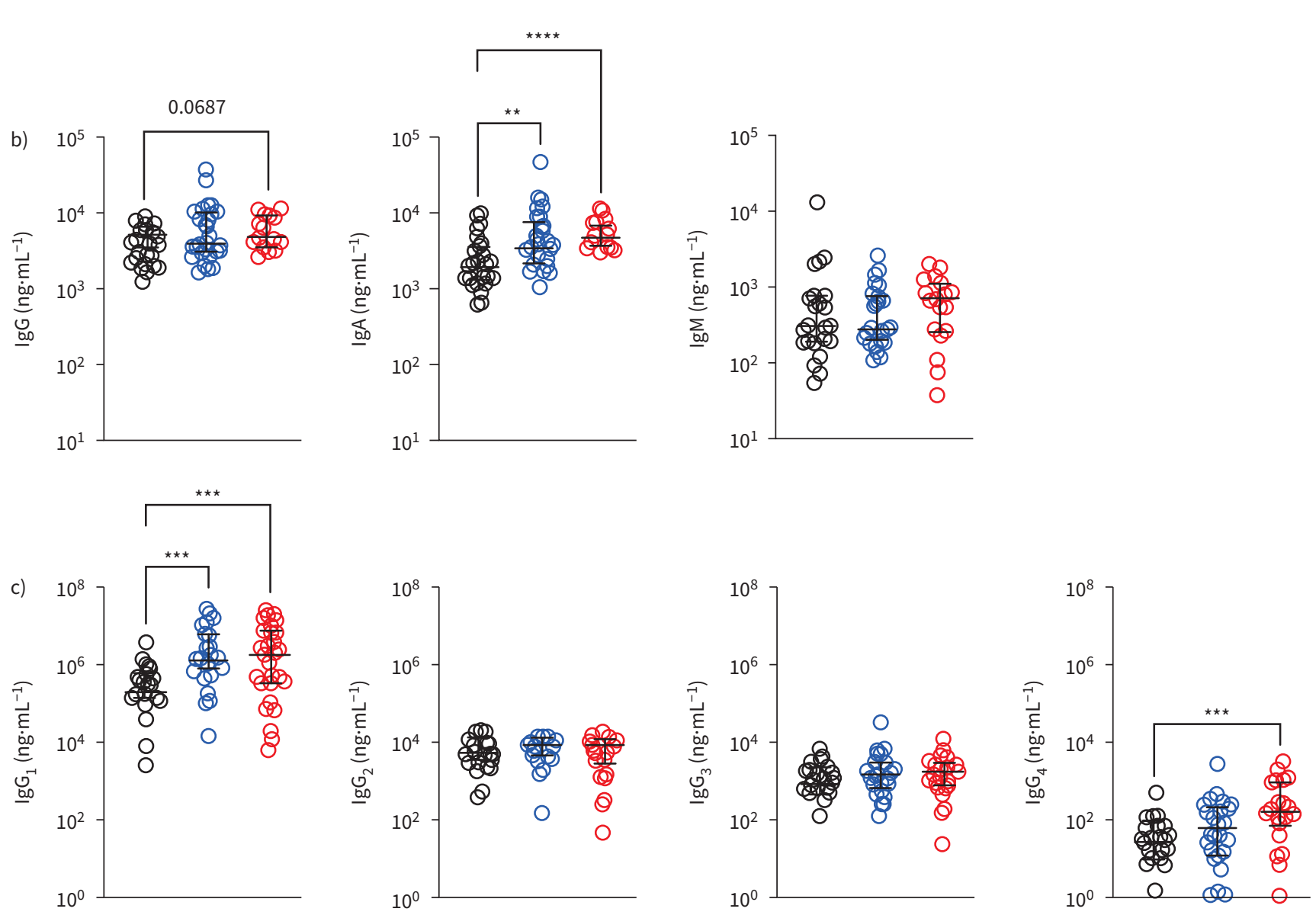

FIGURE 1 Local antibodies are increased in patients with idiopathic pulmonary fibrosis (IPF) and chronic hypersensitivity pneumonitis (CHP). a) Total IgG, IgA and IgM concentrations in plasma from patients with IPF and CHP, and healthy controls. b) Total IgG, IgA and IgM concentrations in bronchoalveolar lavage (BAL) fluid from patients with IPF and CHP, and healthy controls. c) $\lg G_{1}, \operatorname{lgG}_{2}$, IgG $\lg _{3}$ and IgG $\mathrm{I}_{4}$ concentrations in $B A L$ from patients with IPF and CHP, and healthy controls. Each point represents an individual subject. Horizontal bars represent medians and whiskers represent interquartile range. Data were analysed using a non-parametric Kruskal-Wallis test with Dunn's multiple comparison. *: $p<0.05$; ${ }^{\star \star}: p<0.01 ;{ }^{* \star *}: p<0.001 ;{ }^{\star \star \star *}: p<0.0001$.

Patients who formed a distinct cluster based on increased autoantibody score (hereon termed "autoAb") were next tested independently by ELISA for anti-collagen $\mathrm{V}$ and anti-vitronectin autoantibodies, both of which are expressed in the lung tissue $[30,31]$. These autoAb ${ }^{+}$patients also had significantly increased titres of anti-collagen $\mathrm{V}$ and anti-vitronectin autoantibodies in the BAL when determined by ELISA (figure 2e and figure S2A). Importantly, no difference was observed in serum titres of anti-collagen $\mathrm{V}$ and anti-vitronectin antibodies between the AutoAb $b^{-}$and AutoAb ${ }^{+}$matched patients or healthy controls, suggesting that the presence of autoantibodies in these patients was unique to the BAL (figure $2 \mathrm{f}$ and figure S2B). 


\begin{tabular}{|c|c|c|c|c|c|}
\hline Parameter & $\begin{array}{l}\text { Healthy controls } \\
\qquad(n=15)\end{array}$ & $\begin{array}{l}\text { IPF } \\
(n=40)\end{array}$ & $\begin{array}{l}\text { CHP } \\
(n=20)\end{array}$ & $\begin{array}{l}\text { CTD } \\
(n=20)\end{array}$ & $\begin{array}{l}\text { Non-ILD diseased controls } \\
\qquad(\mathrm{n}=5)\end{array}$ \\
\hline Age, years & $61 \pm 5$ & $73 \pm 6$ & $71 \pm 8$ & $70 \pm 12$ & $67 \pm 19$ \\
\hline Female sex & 31 & 33 & 60 & 50 & 20 \\
\hline $\begin{array}{l}\text { Ex-smoker or current } \\
\text { smoker }\end{array}$ & 25 & 68 & 40 & 53 & 80 \\
\hline $\mathrm{FEV}_{1}, \%$ predicted & $101 \pm 14$ & $92 \pm 21$ & $83 \pm 21$ & $86 \pm 28$ & $68 \pm 24$ \\
\hline FVC, \% predicted & $104 \pm 15$ & $91 \pm 21$ & $87 \pm 22$ & $82 \pm 24$ & $91 \pm 22$ \\
\hline$D_{\text {Lco, }} \%$ predicted & $103 \pm 1$ & $55 \pm 15$ & $60 \pm 16$ & $50 \pm 19$ & $53 \pm 16$ \\
\hline Details & NA & NA & NA & NA & $\begin{array}{c}\text { COPD }(n=2) \\
\text { Bronchiectasis }(n=2) \\
\text { Adenocarcinoma }(n=1)\end{array}$ \\
\hline
\end{tabular}

Presence of lower airway autoantibodies is not associated with disease progression and survival Finally, we wanted to determine whether the presence of BAL autoantibodies was associated with more severe disease. Principal component analysis with $k$ means clustering based on IgG autoantibody scores was performed on all patients with fILD,, but not controls and identified three distinct clusters of patients (figure 3a). Clusters 1 and 2 corresponded to subjects who had positive autoantibody signal (figure 3a). There was no difference in lung function between any of the clusters and when correlation analysis was performed, and there were no statistically significant associations between IgG and IgA mean autoantibody scores and lung function parameters (figure $3 b$ and c). Cluster 3 contained a higher percentage of progressors, defined as patients who had a decline in forced vital capacity $>10 \%$ (figure $3 \mathrm{~d}$ ). Cluster 1 had an increased percentage of patients with IPF (56.25\%) and CTD (25\%) compared to clusters 2 and 3, while clusters 2 and 3 had increased proportions of patients with CHP (40.1\% and 33.3\% respectively) (figure 3e). Overall, there was no difference in survival probability between any of the clusters (figure 3f). To further examine the possible role of BAL autoantibodies on IPF disease outcome, a similar cluster analysis was performed, resulting in three clusters of patients (figure S3A). There were no differences in lung function, proportion of progressors or survival probability between any of the clusters (figure S3B-D).

To identify the top putative autoantigens responsible for variance in principal component (PC)1, percentage contributions were analysed. Fibrinogen IV, fibrinogen S, ribophosphoprotein P1, PM.Scl.75, vitronectin and Ro/SSA accounted for the highest contribution to variance in PC1 (figure S4A). Stratification of patients based on presence or absence of autoantibodies against these antigens showed no difference in survival probability (figure S4B). Similarly, for PC2, chondroitin sulfate C, nup 62, heparin, single-stranded RNA, bovine decorin and collagen II had the highest contribution to variance (figure S4C). Stratification of patients based on the presence or absence of these autoantibodies show no significant difference in survival probability (figure S4D). Taken together, these data suggest that while the airways of approximately half of fILD patients are enriched for autoantibodies against cellular and nuclear antigens, these do not associate with disease severity and outcome.

\section{Discussion}

The presence of circulating autoantibodies and their impact on disease outcome in patients with ILD have been widely reported. Here, we sought to address whether patients with ILD have a local airway autoimmune signature, and whether this could be used as a predictor of disease severity and outcome. We show that IgA, IgG 1 and $\operatorname{IgG}_{4}$ are increased in the airways of patients with fILD, and identify a subset of patients with local but not systemic autoantibodies.

Although patients with IPF had modest increases in serum IgG, patients with IPF and CHP both had increases in airway IgG and IgA. In agreement with a previous study, total airway antibody was not associated with worse predicted survival [15]. Specifically, $\operatorname{IgG}_{1}$ was increased in IPF airways while IgG $_{4}$ was increased in IPF and CHP airways. Although IgG is predominantly involved in systemic responses, it is also the most abundant antibody class in the lower respiratory tract $[32,33]$. Unlike IgA and IgM, both 
a)

IgG

$\llbracket$ Healthy $\backsim \mathrm{IPF} \backsim \mathrm{CHP} \backsim \mathrm{CTD} \backsim \mathrm{COPD} \backsim$ Cancer $\backsim$ Bronchiectasis

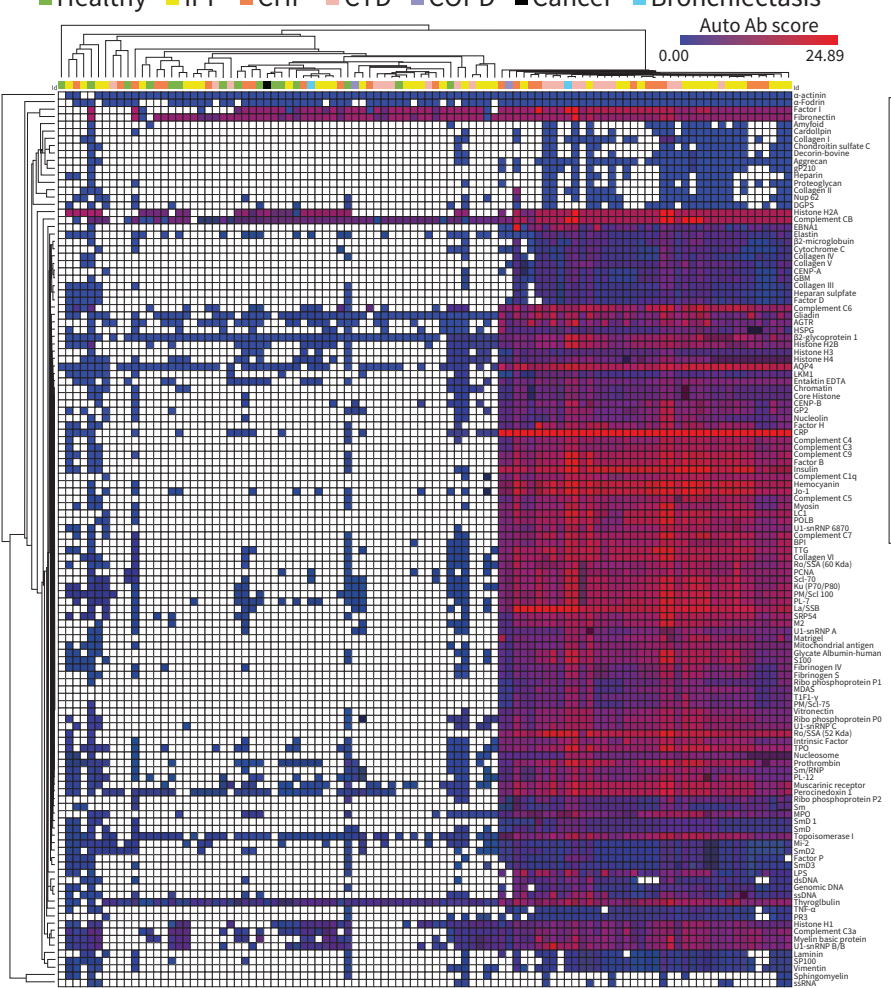

b)

IgA

$\frac{\text { Auto Ab score }}{23.52}$

$\square$ Healthy $\square \mathrm{IPF} \backsim \mathrm{CHP} \backsim \mathrm{CTD} \backsim \mathrm{COPD} \backsim$ Cancer $\backsim$ Bronchiectasis

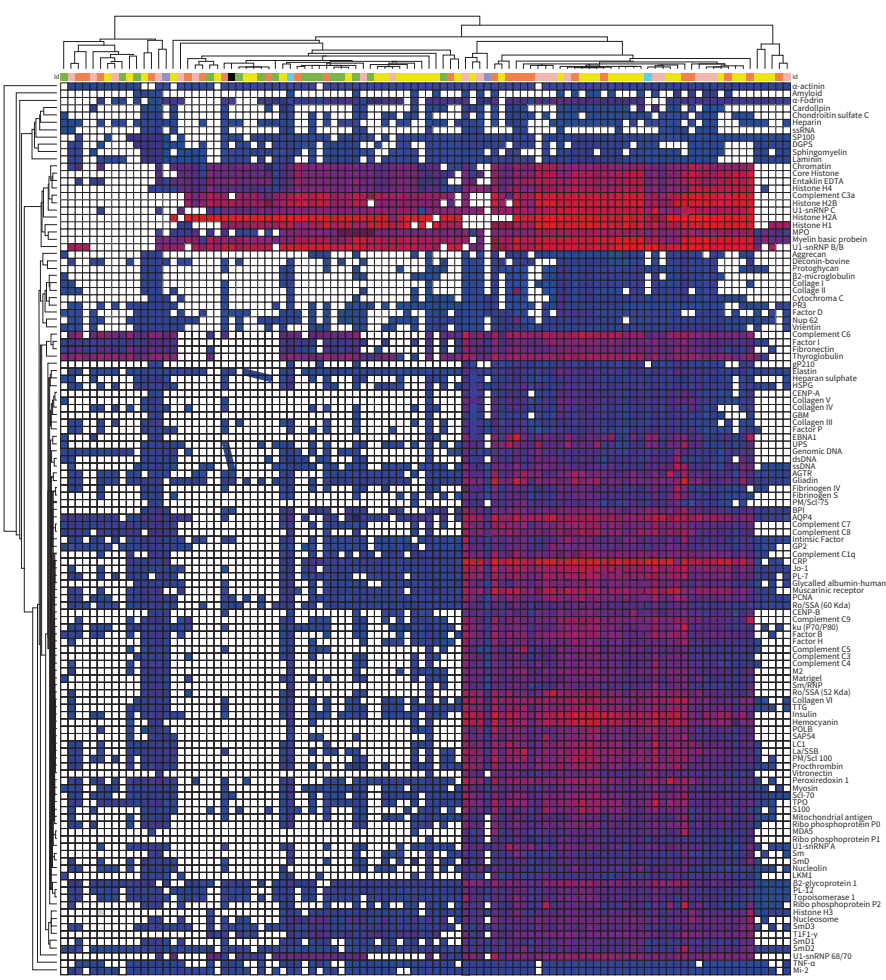

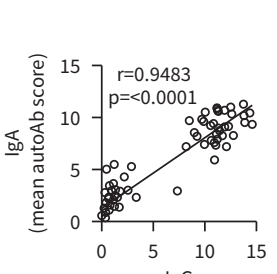

IgG

(mean auto Ab score) d)

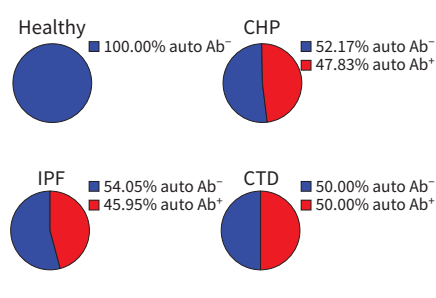

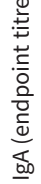

e) Collagen V

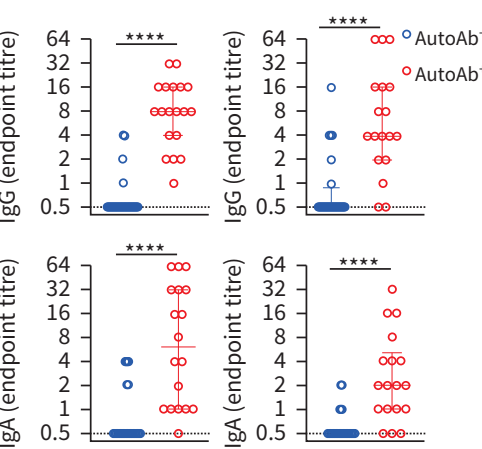

f)

Collagen V

Vitronectin

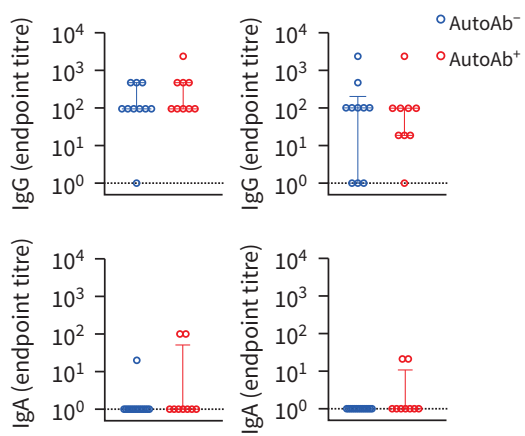

FIGURE 2 IgG and IgA autoantibodies (autoAb) are detectable in the airways of a subset of patients with fibrotic interstitial lung disease. Bronchoalveolar lavage a) IgG and b) IgA autoAb were screened for reactivity against 122 self antigens in idiopathic pulmonary fibrosis (IPF) ( $n=40$ ), chronic hypersensitivity pneumonitis (CHP) $(n=20)$ or connective tissue disease (CTD) patients $(n=20)$ and nonfibrotic controls ( $n=20)$. Heatmaps show autoAb scores, calculated as $\log _{2}$-transformed(net fluorescence intensityxsignal-to-noise ratio+1). White squares indicate samples for which autoantibody signal was not detectable against specific autoantigens. Samples were clustered hierarchically. c) Pearson correlation between mean IgG and IgA autoAb scores. d) Proportions of autoAb-positive individuals in each study group. e) BAL from the array was used to validate the findings independently in a sandwich ELISA assay in order to validate presence of autoantibodies against collagen $V$ and vitronectin. $f$ ) Serum dilutions were also analysed for autoantibodies against collagen $V$ and vitronectin. Each point in $c$, e and $f$ represents an individual subject. Horizontal bars represent medians and whisker represent interquartile ranges. Data in e and $f$ were analysed using a non-parametric MannWhitney U-test. ${ }^{\star \star \star \star}: p<0.0001$.

of which are transcytosed via the polymeric immunoglobulin receptor, IgG is transcytosed bidirectionally via the action of the neonatal Fc receptor [34]. $\operatorname{IgG}_{1}$ is involved in pro-inflammatory responses including complement activation, while $\operatorname{IgG}_{4}$ suppresses immune activation and promotes tolerance [35]. IgA was 

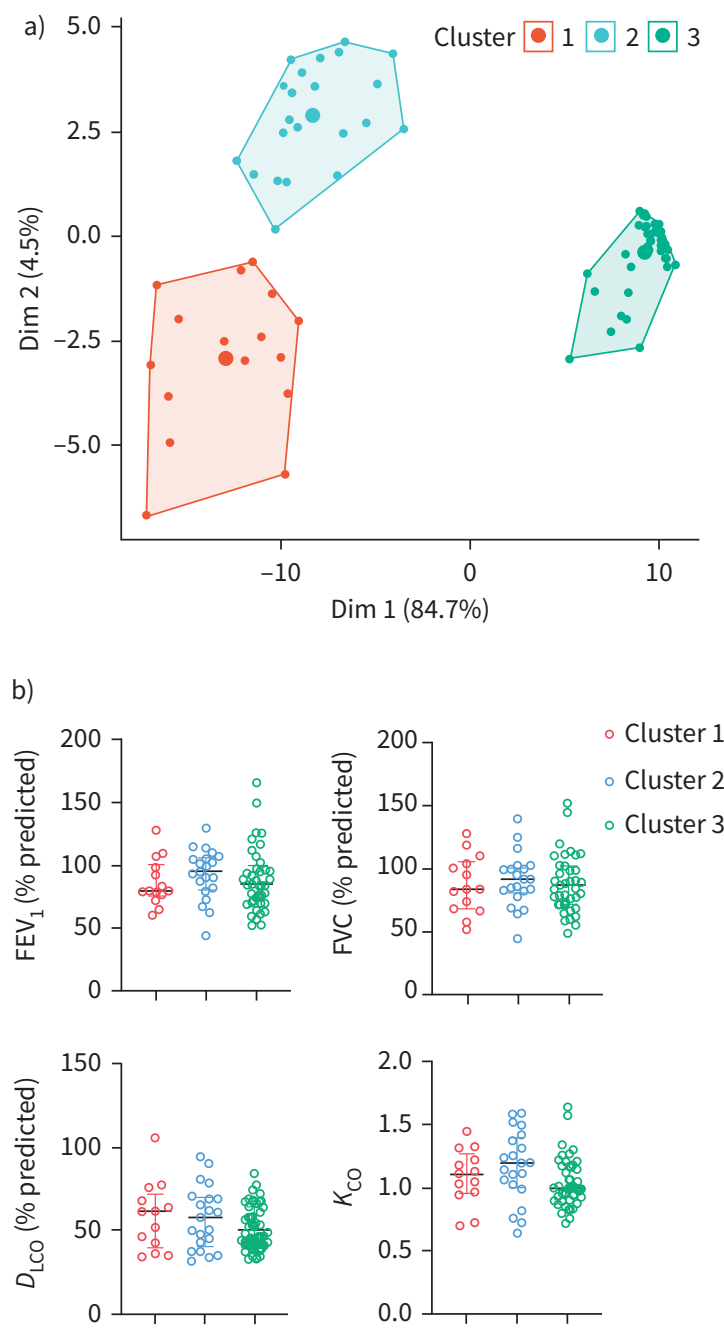

c)

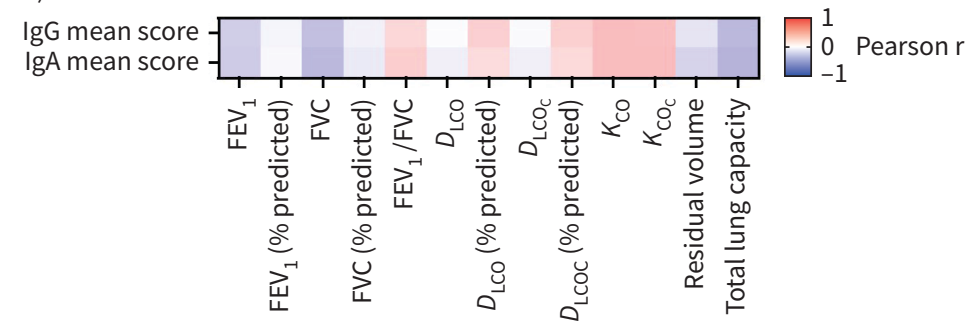

d)

e)

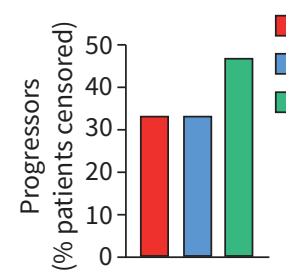

Cluster 1

Cluster 2

Cluster 3

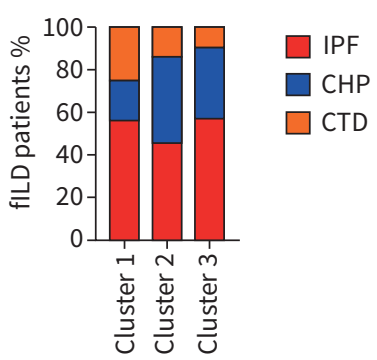

f)

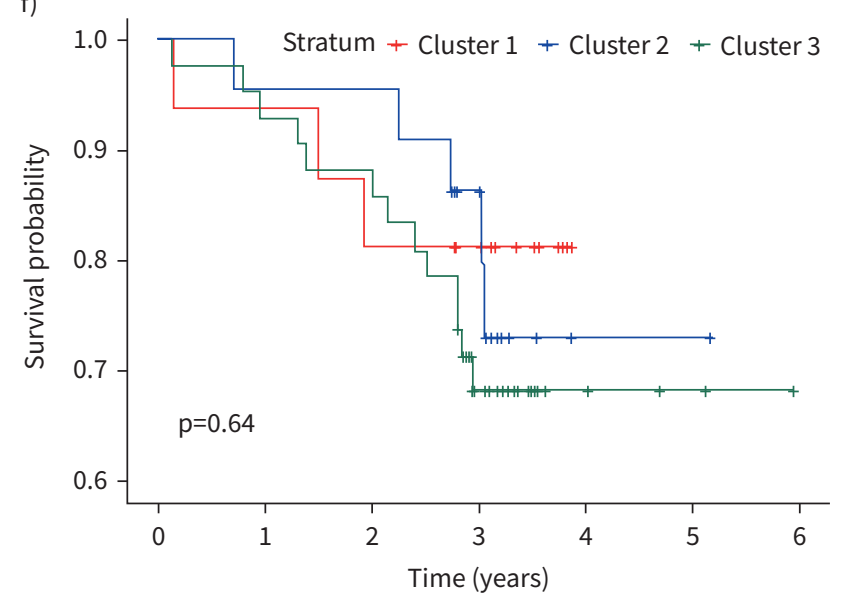

Number at risk

\begin{tabular}{|c|c|c|c|c|c|c|c|}
\hline \multirow{4}{*}{ 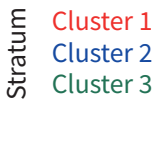 } & 16 & 15 & 13 & 10 & 0 & 0 & 0 \\
\hline & 22 & 21 & 21 & 14 & 1 & 1 & 0 \\
\hline & 42 & 39 & 37 & 20 & 4 & 2 & 0 \\
\hline & 0 & 1 & 2 & 3 & 4 & 5 & 6 \\
\hline
\end{tabular}

FIGURE 3 Presence of airway autoantibody in patients with fibrotic interstitial lung disease (fILD) is not associated with reduced lung function or survival probability. a) Principal component analysis followed by $k$ means clustering based on airway IgG autoantibody scores. b) Patient lung function scores by cluster. c) Pearson correlation between mean IgG and IgA autoantibody scores and lung function parameters. d) Percentage of patients who show lung function decline as a proportion of all patients censored by cluster. e) Proportions of individuals with idiopathic pulmonary fibrosis (IPF), chronic hypersensitivity pneumonitis (CHP) and connective tissue disease (CTD) within each cluster. f) Kaplan-Meier curve generated using the Cox proportional hazards model showing predicted survival probability in patients in each of the clusters. Each point in a and b represents an individual subject. Horizontal bars represent medians and whiskers represent interquartile ranges. Dim: dimension; FEV ${ }_{1}$ : forced expiratory volume in $1 \mathrm{~s}$; FVC: forced vital capacity; $D_{\mathrm{Lco}}$ : diffusing capacity of the lung for carbon monoxide: $K_{\mathrm{co}}$ : transfer coefficient of the lung for carbon monoxide; $D_{\mathrm{LCO}}: D_{\mathrm{LCO}}$ corrected for haemoglobin; $K_{\mathrm{CO}_{c}}: K_{\mathrm{CO}}$ corrected for haemoglobin.

also increased in IPF and CHP airways. In the gut, IgA is responsible for maintaining homeostatic relationships with commensals in the gastrointestinal tract through immune exclusion and bacterial enchainment [36]. However, its role in the lower respiratory tract remains to be defined. Mucosal IgG $_{1}$ also plays an important role in maintaining commensals [32, 35]. The lower airway microbiomes in IPF and 
CHP are distinct from each other, and both different from that of healthy controls [24, 37-39]. The increase in IgA and IgG $_{1}$ seen in the airways of patients with IPF and CHP may well be a compensatory reaction to this microbial dysbiosis. Further work will be needed to understand these relationships and their effects on disease outcomes.

Over the last decade, studies have begun to explore the role of autoantigens in fILD pathology. However, the relationship between systemic autoimmune disease and ILD remains poorly understood. One of the first circulating epithelial autoantibodies to be identified in pulmonary fibrosis was anti-cytokeratin 8 and this provided evidence to support the hypothesis of autoantibody-mediated lung injury [40]. Since then, multiple circulating autoantibodies have been reported against human epithelial and extracellular matrix proteins, including annexin 1 , heat shock protein 70 , vimentin and periplakin, some of these correlating with disease severity $[4,5,15,20,41]$.

We confirm and extend previous analyses of autoantibodies in fILD and show for the first time by large-scale array that autoantibodies against a range of matrix components are also detectable locally within the airways of a subset of patients with fibrotic lung disease. These data suggests that there is a specific local autoimmune signature in some patients with fibrotic lung disease. Importantly, these data also support a link between autoimmunity and tissue damage and fibrosis. Early studies showed that patients with fibrosing alveolitis have increased concentrations of IgG autoantibodies to proteins expressed within lung tissue [42]. Sнum and co-workers [18, 23] elegantly showed that autoantibody targeting of BPIFB1, a protein expressed exclusively in the lung tissue, results in the development of lung fibrosis in a murine model.

Here, we show that patients have autoantibodies against a range of proteins expressed within the lung, some which may be associated with tissue damage, such as nuclear proteins. Indeed, autoantibodies against some of the antigens in our array, such as vimentin, have been previously reported in fILDs [4, 16]. This broad pattern of autoantibody specificity has also been reported in the serum from severe COPD patients and was particularly associated with emphysema, suggesting that autoantibody profiles differ between disease groups [8]. Interestingly, most of the autoantibodies we present here are against proteins that are often upregulated in fILD. Vitronectin is significantly increased in the BAL of patients with ILD [31]. Similarly, many of the components of the extracellular matrix, such as the collagen proteins, particularly collagen I and V, are overexpressed in the wound repair process. Collagen V plays a central role in the formation of fibrillar collagen mesh and is also involved in regulating the fibre size [43]. In parenchymal fibrosis, collagen $\mathrm{V}$ is highly overexpressed in IPF lungs and much of the morphological disorganisation of fibrillar collagen can be attributed to this aberrant expression of collagen V [19, 43]. Interestingly, it has been reported that $40-60 \%$ of patients with IPF have autoimmune responses against collagen V, similar to the proportions observed in the current study [19]. It is possible that increased expression of collagen $\mathrm{V}$ in lung fibrosis results leads to an aberrant humoral response against an otherwise innocuous protein. A recent phase I clinical trial involving oral immunotherapy with bovine collagen $\mathrm{V}$ in patients with IPF led to stabilised lung function and reduced matrix metalloproteinase expression, possibly through the induction of humoral tolerance [30].

The site of autoantibody production in fILD remains elusive. The presence of a local airway, but not a systemic, autoimmune signature suggests that the production of autoantibodies may be occurring locally. Early studies by RANGEL-Moreno et al. [44] showed that autoantibody production can occur locally within lymphocyte aggregates in fibrotic lung tissue. Specifically, they showed that autoantibodies against both vimentin and citrullinated proteins localise around B-cell aggregates within the fibrotic lung tissue [16, 44]. Indeed, B-cell aggregates are observed in biopsy tissue from human IPF lungs [45]. Local autoantibody production is also supported by tissue-resident B-cells in other organs, such as the upper respiratory tract and synovia in chronic rhinosinusitis with nasal polyps and rheumatoid arthritis, respectively [46, 47]. It is important to note that lymphoid aggregates are also not observed in all patients with rheumatoid arthritis-associated lung fibrosis [44]. This may explain why only some patients develop a local autoimmune signature. However, our findings suggest that the link between local autoantibody and disease progression cannot be assumed, and may also depend on other factors, such as time of sampling. One of the main limitations of the study is that the median follow-up time was 3 years, and that may limit our ability to detect differences in survival between those patients with and without local autoantibodies. However, there is no signal of rapid deterioration. Since none of the patients in our cohort were on immunosuppression at the time of the bronchoscopy, further work is also required to determine whether immunosuppressive treatments targeting the humoral immune response (e.g. rituximab) can affect local autoantibody signatures and subsequent disease outcome. 
We have demonstrated, for the first time, that there is a broad spectrum of autoantibodies present locally within the airways of patients with fILD that is not present in the circulation, supporting our hypothesis that local antibody-mediated tissue damage can drive the pathology of lung fibrosis. Subsequent investigations should therefore aim to define the role of these autoantibodies in tissue damage. Ultimately, if humoral dysregulation is a key player in severe disease, then a number of targeted therapies may be more beneficial to outcome than nonspecific treatments.

Provenance: Submitted article, peer reviewed.

Conflict of interest: K. Boustani has nothing to disclose. P. Ghai has nothing to disclose. R. Invernizzi has nothing to disclose. R.J. Hewitt has nothing to disclose. T.M. Maher reports grants and personal fees from GSK, personal fees from Boehringer Ingelheim, grants and personal fees from AstraZeneca, personal fees from Roche, Galapagos, Celgene, Pliant, Blade Therapeutics, Respivant, Bristol-Myers Squibb, Galecto, Theravance, IQVIA and Veracyte, outside the submitted work. Q-Z. Li has nothing to disclose. P.L. Molyneaux reports receiving grant funding to institution from AstraZeneca, outside the submitted work; and consulting speaker fees from Boehringer Ingelheim and Hoffman-La Roche, outside the submitted work. J.A. Harker has nothing to disclose.

Support statement: This work was funded by a Rosetrees Seed Fund to P.L. Molyneaux and J.A. Harker (A2172). K. Bustani was funded by an Asthma UK studentship to J.A. Harker and P.L. Molyneaux as part of the Asthma UK Centre in Allergic Mechanisms of Asthma (AUK-BC-2015-01). Funding information for this article has been deposited with the Crossref Funder Registry.

\section{References}

1 Lederer DJ, Martinez FJ. Idiopathic pulmonary fibrosis. N Engl J Med 2018; 378: 1811-1823.

2 Kreuter M, Bonella F, Wijsenbeek M, et al. Pharmacological treatment of idiopathic pulmonary fibrosis: current approaches, unsolved issues, and future perspectives. Biomed Res Int 2015; 2015: 329481.

3 Allden SJ, Ogger PP, Ghai P, et al. The transferrin receptor CD71 delineates functionally distinct airway macrophage subsets during idiopathic pulmonary fibrosis. Am J Respir Crit Care Med 2019; 200: 209-219.

4 Li FJ, Surolia R, Li H, et al. Autoimmunity to vimentin is associated with outcomes of patients with idiopathic pulmonary fibrosis. J Immunol 2017; 199: 1596-1605.

5 Kahloon RA, Xue J, Bhargava A, et al. Patients with idiopathic pulmonary fibrosis with antibodies to heat shock protein 70 have poor prognoses. Am J Respir Crit Care Med 2013; 187: 768-775.

6 Feghali-Bostwick CA, Wilkes DS. Autoimmunity in idiopathic pulmonary fibrosis are circulating autoantibodies pathogenic or epiphenomena? Am J Respir Crit Care Med 2011; 183: 691-692.

7 Lee JS, Kim EJ, Lynch KL, et al. Prevalence and clinical significance of circulating autoantibodies in idiopathic pulmonary fibrosis. Respir Med 2013; 107: 249-255.

8 Packard TA, Li QZ, Cosgrove GP, et al. COPD is associated with production of autoantibodies to a broad spectrum of self-antigens, correlative with disease phenotype. Immunol Res 2013; 55: 48-57.

9 Feghali-Bostwick CA, Gadgil AS, Otterbein LE, et al. Autoantibodies in patients with chronic obstructive pulmonary disease. Am J Respir Crit Care Med 2008; 177: 156-163.

10 Feghali-Bostwick CA, Tsai CG, Valentine VG, et al. Cellular and humoral autoreactivity in idiopathic pulmonary fibrosis. J Immunol 2007; 179: 2592-2599.

11 Keir GJ, Maher TM, Ming D, et al. Rituximab in severe, treatment-refractory interstitial lung disease. Respirology 2014; 19: 353-359.

12 Lota HK, Keir GJ, Hansell DM, et al. Novel use of rituximab in hypersensitivity pneumonitis refractory to conventional treatment. Thorax 2013; 68: 780-781.

13 Keir GJ, Maher TM, Hansell DM, et al. Severe interstitial lung disease in connective tissue disease: Rituximab as rescue therapy. Eur Respir J 2012; 40: 641-648.

14 Saul M, Raval JS, Duncan SR, et al. Autoantibody-targeted treatments for acute exacerbations of idiopathic pulmonary fibrosis. PLOS ONE 2015; 10: e0127771.

15 Mills R, Mathur A, Nicol LM, et al. Intrapulmonary autoantibodies to HSP72 are associated with improved outcomes in IPF. J Immunol Res 2019; 2019: 1845128.

16 Kinloch AJ, Kaiser $\mathrm{Y}$, Wolfgeher $\mathrm{D}$, et al. In situ humoral immunity to vimentin in $\mathrm{HLA}-\mathrm{DRB} 1^{\star} 03^{+}$patients with pulmonary sarcoidosis. Front Immunol 2018; 9: 1516.

17 Hoyne GF, Elliott H, Mutsaers SE, et al. Idiopathic pulmonary fibrosis and a role for autoimmunity. Immunol Cell Biol 2017; 95: 577-583.

18 Shum AK, Alimohammadi M, Tan CL, et al. BPIFB1 is a lung-specific autoantigen associated with interstitial lung disease. Sci Transl Med 2013; 5: 206ra139.

19 Vittal R, Mickler EA, Fisher AJ, et al. Type V collagen induced tolerance suppresses collagen deposition, TGF-beta and associated transcripts in pulmonary fibrosis. PLOS ONE 2013; 8: e76451. 
20 Taillé C, Grootenboer-Mignot S, Boursier C, et al. Identification of periplakin as a new target for autoreactivity in idiopathic pulmonary fibrosis. Am J Respir Crit Care Med 2011; 183: 759-766.

21 Nakos G, Adams A, Andriopoulos N. Antibodies to collagen in patients with idiopathic pulmonary fibrosis. Chest 1993; 103: 1051-1058.

22 Alimohammadi M, Dubois N, Skoldberg F, et al. Pulmonary autoimmunity as a feature of autoimmune polyendocrine syndrome type 1 and identification of KCNRG as a bronchial autoantigen. Proc Natl Acad Sci USA 2009; 106: 4396-4401.

23 Shum AK, Devoss J, Tan CL, et al. Identification of an autoantigen demonstrates a link between interstitial lung disease and a defect in central tolerance. Sci Transl Med 2009; 1: 9ra20.

24 Invernizzi R, Wu BG, Barnett J, et al. The respiratory microbiome in chronic hypersensitivity pneumonitis is distinct from that of idiopathic pulmonary fibrosis. Am J Respir Crit Care Med 2020; 203: 339-347.

25 Molyneaux PL, Smith JJ, Saunders P, et al. BAL is safe and well tolerated in individuals with idiopathic pulmonary fibrosis: an analysis of the PROFILE Study. Am J Respir Crit Care Med 2021; 203: 136-139.

26 Zhu H, Luo H, Yan M, et al. Autoantigen microarray for high-throughput autoantibody profiling in systemic lupus erythematosus. Genomics Proteomics Bioinformatics 2015; 13: 210-218.

27 Goutaki M, Pescatore AM, Singh P, et al. Increased prevalence of pre-school wheeze is not explained by time trends in body mass index. Eur Respir J 2014; 44: 1078-1082.

28 Low TB, Greene CM, O'Neill SJ, et al. Quantification and evaluation of the role of antielastin autoantibodies in the emphysematous lung. Pulm Med 2011; 2011: 826160.

29 Wen L, Krauss-Etschmann S, Petersen F, et al. Autoantibodies in chronic obstructive pulmonary disease. Front Immunol 2018; 9: 66.

30 Wilkes DS, Chew T, Flaherty KR, et al. Oral immunotherapy with type V collagen in idiopathic pulmonary fibrosis. Eur Respir J 2015; 45: 1393-1402.

31 Pohl WR, Conlan MG, Thompson AB, et al. Vitronectin in bronchoalveolar lavage fluid is increased in patients with interstitial lung disease. Am Rev Respir Dis 1991; 143: 1369-1375.

32 Cerutti A, Chen K, Chorny A. Immunoglobulin responses at the mucosal interface. Annu Rev Immunol 2011; 29: 273-293.

33 Peebles RS, Liu MC, Lichtenstein LM, et al. IgA, IgG and IgM quantification in bronchoalveolar lavage fluids from allergic rhinitis, allergic asthmatics, and normal subjects by monoclonal antibody-based immunoenzymatic assays. J Immunol Methods 1995; 179: 77-86.

34 Dickinson BL, Badizadegan $\mathrm{K}$, Wu Z, et al. Bidirectional FcRn-dependent IgG transport in a polarized human intestinal epithelial cell line. J Clin Invest 1999; 104: 903-911.

35 Vidarsson G, Dekkers G, Rispens T. IgG subclasses and allotypes: from structure to effector functions. Front Immunol 2014; 5: 520 .

36 Moor K, Diard M, Sellin ME, et al. High-avidity IgA protects the intestine by enchaining growing bacteria. Nature 2017; 544: 498-502.

37 Molyneaux PL, Cox MJ, Wells AU, et al. Changes in the respiratory microbiome during acute exacerbations of idiopathic pulmonary fibrosis. Respir Res 2017; 18: 29.

38 Molyneaux PL, Cox MJ, Willis-Owen SAG, et al. The role of bacteria in the pathogenesis and progression of idiopathic pulmonary fibrosis. Am J Respir Crit Care Med 2014; 190: 906-913.

39 O'Dwyer DN, Ashley SL, Gurczynski SJ, et al. Lung microbiota contribute to pulmonary inflammation and disease progression in pulmonary fibrosis. Am J Respir Crit Care Med 2019; 199: 1127-1138.

40 Dobashi N, Fujita J, Yuji O, et al. Detection of anti-cytokeratin 8 antibody in the serum of patients with cryptogenic fibrosing alveolitis and pulmonary fibrosis associated with collagen vascular disorders. Thorax 1998; 53: 969-974.

41 Kurosu K, Takiguchi $\mathrm{Y}$, Okada $\mathrm{O}$, et al. Identification of annexin 1 as a novel autoantigen in acute exacerbation of idiopathic pulmonary fibrosis. J Immunol 2008; 181: 756-767.

42 Wallace WA, Roberts SN, Caldwell H, et al. Circulating antibodies to lung protein(s) in patients with cryptogenic fibrosing alveolitis. Thorax 1994; 49: 218-224.

43 Parra ER, Teodoro WR, Velosa AP, et al. Interstitial and vascular type V collagen morphologic disorganization in usual interstitial pneumonia. J Histochem Cytochem 2006; 54: 1315-1325.

44 Rangel-Moreno J, Hartson L, Navarro C, et al. Inducible bronchus-associated lymphoid tissue (iBALT) in patients with pulmonary complications of rheumatoid arthritis. J Clin Invest 2006; 116: 3183-3194.

45 Xue J, Kass DJ, Bon J, et al. Plasma B lymphocyte stimulator and B cell differentiation in idiopathic pulmonary fibrosis patients. J Immunol 2013; 191: 2089-2095.

46 Humby F, Bombardieri M, Manzo A, et al. Ectopic lymphoid structures support ongoing production of class-switched autoantibodies in rheumatoid synovium. PLoS Med 2009; 6: e1.

47 Song J, Wang $\mathrm{H}$, Zhang YN, et al. Ectopic lymphoid tissues support local immunoglobulin production in patients with chronic rhinosinusitis with nasal polyps. J Allergy Clin Immunol 2018; 141: 927-937. 